\section{Psychosis and drug dependence:}

\section{results from a national survey of prisoners}

\author{
M. FARRELL, A. BOYS, P. BEBBINGTON, T. BRUGHA, J. COID, R. JENKINS, \\ G. LEWIS, H. MELTZER, J. MARSDEN, N. SINGLETON and C. TAYLOR
}

Severe mental illness and drug dependence are a major burden within the criminal justice system and within the general health services. The links between drug use and psychosis are therefore of significant aetiological and prognostic interest. It is difficult to study this relationship outside of clinical populations, as both psychosis and severe drug dependence are uncommon. However, national data on psychiatric morbidity in prisoners suggest that within this population psychosis and drug dependence are relatively widespread (Singleton et al, 1998). The prevalence of psychosis and psychotic symptoms (i.e. having some symptoms but not meeting criteria for association as functional psychosis) in the Office for National Statistics (ONS) household and prison surveys have been compared elsewhere (further details available from the author upon request). Although no differences were found in the range of psychotic symptoms exhibited by the two groups, estimated rates of functional psychosis were over 10 times greater in the prison survey $(52 / 1000 v .4 .5 / 1000$; Singleton et al, 1998). This paper examines the drug use and dependence characteristics of prisoners in England and Wales classified with functional psychosis.

\section{METHOD}

\section{Sample}

All 131 prisons operational at the time of the survey agreed to participate. The survey was implemented in two stages (see Singleton $e$ al, 1998 for a complete description of the survey methods and protocol). The Local Inmate Directory System (a database of information on all current prisoners and held by all prisons) was used as the sampling frame. The sampling fractions used were: 1 in 34 male sentenced prisoners; 1 in 8 male remand prisoners; and 1 in 3 female prisoners (both on remand and sentenced). Of the resulting
3563 selected prisoners, 3142 (88.2\%) completed a face-to-face interview with trained ONS staff. Thirty-seven prisoners agreed to participate but did not complete the lengthy questionnaire; a further $5.6 \%$ $(n=198)$ refused to participate and 118 prisoners could not be contacted when the interview was scheduled to take place. Language difficulties excluded 53 inmates and 15 were judged to be too dangerous or disturbed to be interviewed. In the second stage, 1 in 5 interviewees $(n=661)$ was invited to participate in a further detailed interview containing the Schedules for Clinical Assessment in Neuropsychiatry (SCAN version 1.0; World Health Organization, 1992). This interview was administered by trained, clinically qualified staff. This paper reports on data collected from $503(76.1 \%)$ of these second-stage respondents. Fifty prisoners $(7.6 \%)$ refused to participate and the remainder could not be contacted. Accordingly, the present analysis is based only on these 503 respondents, which is a random sub-sample of the prison survey sample.

\section{Measures}

The interview included socio-demographic descriptors, daily living and social functioning characteristics, stressful life events, general health and service utilisation, as well as psychiatric morbidity. Background variables (such as age and ethnicity) were measured using a series of closed questions. Questions also addressed criminal history and the nature of particular stressful or traumatic life experiences or events (such as homelessness or being in local authority care as a child).

\section{Psychotic disorder}

The presence of functional psychosis during the year before the interview was assessed using diagnostic criteria for ICD-10 derived algorithmically from the SCAN schedule (World Health Organization, 1992). Each type of psychotic phenomenon (symptom) was rated individually in SCAN. As part of SCAN, clinicians rated whether such a phenomenon was attributable to acute toxic or withdrawal effects of alcohol or drug use. Codes that cover psychosis categories were used. Respondents who were classed in any of the categories F20-31 and F32-33 were grouped in the category of 'any functional psychosis'. Thus, all prisoners who met diagnostic criteria for psychotic disorder, including 
schizophrenia, affective psychosis and drug-induced psychosis were included in our analyses.

\section{Substance use}

In addition to questions on alcohol and cigarettes, respondents were asked to indicate which of a list of eight substances (including solvents) they had ever used. For each drug, the age at first use was recorded together with an estimate of the total number of instances of use. For the purposes of the current analyses, this information was then transformed into binary variables to indicate first use before the age of 16 years and more than 100 occasions of use.

\section{Dependence measures}

Drug dependence was assessed using questions based on the Diagnostic Interview Schedule (Robins \& Regier, 1991). Dependence on cannabis, amphetamine, cocaine, heroin and non-prescribed methadone was measured using five items: (a) daily use for 2 weeks or more; (b) a sense of need or dependence; (c) an inability to abstain; (d) tolerance; (e) withdrawal symptoms. The response period for these questions was the 12 months before starting the current prison term. These questions have also been used in the household survey on psychiatric morbidity in Great Britain (Meltzer et al, 1995), and were asked of the total $(n=3142)$ prison sample (Singleton et al, 1999). In previous studies, for drugs other than cannabis, the ONS has used a positive response to any of the five items to indicate dependence. It has been suggested that the first item does not separate frequent recreational users of cannabis from 'dependent' individuals (Singleton et al, 1998). Consequently a threshold of at least two positive responses was used to indicate cannabis dependence; a threshold of at least four positive responses to the five items was used as a criterion for 'severe' dependence.

Hazardous alcohol use and dependence were measured using the Alcohol Use Disorders Identification Test (AUDIT; Babor et al, 1992). The AUDIT has good sensitivity and specificity in addition to concurrent and predictive validity (Claussen \& Aasland, 1993; Bohn et al, 1995; Conigrave et al, 1995). Although the AUDIT questionnaire usually assesses alcohol use during the past 12 months, to harmonise recall with the drug dependence measures a response period of the 12 months before entering prison was used.

Items on smoking were adapted from those used in the Survey of the Physical Health of Prisoners in 1994 (Bridgwood \& Malbon, 1995) and the Survey of Psychiatric Morbidity among adults living in private households (Meltzer et al, 1995). One item (perceived difficulty of not smoking for a day, rated on a four-point scale) was used to indicate the extent of nicotine dependence.

\section{RESULTS}

\section{Sample characteristics}

Of the 503 prisoners who participated, 394 $(78.3 \%)$ were male and the majority $(418$; $83.1 \%)$ described their ethnic origin as 'White'. Sixty per cent of the sample were convicted and sentenced and $29.8 \%$ were on remand. Two-fifths $(202 ; 40.2 \%)$ were in prison for the first time. The rest reported between 1 and 30 previous prison sentences (mean $=2.4)$.

Approximately $10 \%$ (48 participants) of the study sample was classified through SCAN interview as having 'any functional psychosis' during the past year (this being the unadjusted percentage). Table 1 summarises the key characteristics of the respondents and presents odds ratios (ORs) for functional psychosis, together with corresponding probability values and $95 \%$ confidence intervals.

There were few significant relationships between background variables and functional psychosis. Prisoners aged over 40 years were less likely to have been classified with functional psychosis than those aged 16-20 $(\mathrm{OR}=0.11, P<0.05)$. Those who reported that they had been homeless at some point in their lives were more than twice as likely to have this classification than others $(\mathrm{OR}=2.64, P<0.01)$. Similarly, inmates who had been in the care of the local authorities at some point before the age of 16 were twice as likely to have functional psychosis $(\mathrm{OR}=1.99, P<0.05)$.

\section{Psychoactive substance use}

Most of the sample (417 participants; $82.9 \%$ ) were current cigarette smokers and smoked an average of 15.4 cigarettes a day (range 1-80). The risk of functional psychosis was positively related to the perceived difficulty of not smoking for a day $(\mathrm{OR}=1.44, P<0.01)$.
Seventy prisoners $(13.9 \%)$ had not drunk alcohol during the year before entering prison and so did not complete the questions on alcohol use. Among the drinkers, there was no evidence for a significant relationship between AUDIT scores and functional psychosis (OR for AUDIT score of $>16=1.56$; NS).

Inmates who had first used cannabis, amphetamine, opiates or cocaine before the age of 16 were at greater risk of functional psychosis. In particular, early cocaine initiators were 5.5-times more likely to have this classification. Early users of cannabis were twice as likely to suffer from psychosis, and for amphetamines the odds were tripled.

Just under a third of the sample (164 participants; $32.6 \%$ ) reported that they had used at least one stimulant drug (i.e. amphetamines, cocaine powder or crack cocaine) on over 100 separate occasions. Frequent users of these drugs were at greater risk of functional psychosis than those who had used less extensively. For example, for those who had used at least one stimulant on over 100 occasions, the risk of functional psychosis was more than double that of other interviewees $(\mathrm{OR}=2.25, P<0.01)$.

Just over two-fifths $(214 ; 42.5 \%)$ of the inmates were classified as drug-dependent and $146(29 \%)$ reached the criteria for 'severe' dependence for at least one drug. Dependence and severe dependence were a significant risk factor for functional psychosis for all drug types, with the exception of heroin. The greatest risk was associated with severe cocaine dependence $(\mathrm{OR}=8.51, P<0.001)$, followed by severe dependence on any stimulant drug $(\mathrm{OR}=6.24, P<0.001)$. Those classified as severely dependent on cannabis were also almost five times more likely to have a classification of functional psychoses $(\mathrm{OR}=4.77, P<0.001)$.

\section{Logistic regression}

The relationship between dependence measures (dependence and severe dependence) and functional psychosis, while controlling for other variables listed in Table 1, was examined by logistic regression analysis. Table 2 summarises the final model obtained (adjusted ORs, followed by $P$ values and $95 \%$ confidence intervals). The background variables had no significant direct relationship with psychosis when the level of dependence was controlled. 
Table I Sample characteristics together with odds ratios for functional psychosis $(n=503)$

\begin{tabular}{|c|c|c|c|c|c|}
\hline & Frequency (\%) & $\%$ with psychosis & Odds ratio & $P$ & $95 \% \mathrm{Cl}$ \\
\hline \multicolumn{6}{|l|}{ Gender } \\
\hline Male & $394(78.3)$ & 8.4 & 1.00 & - & - \\
\hline Female & $109(21.7)$ & 13.8 & 1.75 & 0.094 & $0.91-3.35$ \\
\hline \multicolumn{6}{|l|}{ Ethnic group } \\
\hline White & $418(83.0)$ & 10.1 & 1.00 & - & - \\
\hline Black & $60(11.9)$ & 8.3 & 0.81 & 0.677 & $0.3 \mathrm{I}-2.15$ \\
\hline Other & $25(5.0)$ & 4.0 & 0.37 & 0.340 & $0.05-2.83$ \\
\hline \multicolumn{6}{|l|}{ Age group } \\
\hline $16-20$ & $101(20.0)$ & 11.9 & 1.00 & - & - \\
\hline $21-29$ & $206(40.9)$ & 10.2 & 0.84 & 0.654 & $0.40-1.79$ \\
\hline $30-39$ & $127(25.2)$ & 11.0 & 0.92 & 0.840 & $0.40-2.08$ \\
\hline $40+$ & $69(13.7)$ & 1.5 & 0.11 & 0.035 & $0.0 \mathrm{I}-0.86$ \\
\hline Ever been homeless & $206(41.0)$ & 14.6 & 2.64 & 0.002 & $1.43-4.88$ \\
\hline In local authority care as a child & $140(27.8)$ & 14.3 & 1.99 & 0.027 & $1.08-3.67$ \\
\hline Sentenced at time of interview & $30 \mathrm{I}(59.8)$ & 8.6 & 0.77 & 0.400 & $0.43-1.41$ \\
\hline First time in prison & $202(40.2)$ & 9.0 & 1.18 & 0.594 & $0.65-2.15$ \\
\hline \multicolumn{6}{|l|}{ Substance use variables } \\
\hline \multicolumn{6}{|l|}{ Audit score } \\
\hline $0-7$ & $221(43.9)$ & 8.1 & 1.00 & - & - \\
\hline $8-15$ & $134(26.6)$ & 9.0 & I.II & 0.266 & $0.52-2.38$ \\
\hline $16+$ & $148(29.4)$ & 12.2 & 1.56 & 0.205 & $0.78-3.11$ \\
\hline Perceived difficulty of not smoking & 2.38 & - & $\mathrm{I} .44$ & 0.003 & $1.13-1.83$ \\
\hline \multicolumn{6}{|l|}{ Early use } \\
\hline First cannabis use before 16 years & $222(44.1)$ & I3.I & 2.07 & 0.019 & $1.13-3.80$ \\
\hline First amphetamine use before 16 years & $79(15.7)$ & 20.3 & 3.11 & 0.001 & $1.61-6.00$ \\
\hline First cocaine/crack use before 16 years & $38(7.6)$ & 31.6 & 5.50 & $<0.00 \mathrm{I}$ & $2.56-11.8$ \\
\hline First opiate use before 16 years & $39(7.8)$ & 20.5 & 2.73 & 0.019 & $1.17-6.34$ \\
\hline \multicolumn{6}{|l|}{ Frequency of use } \\
\hline Used at least one stimulant over 100 times & $164(32.6)$ & 14.6 & 2.25 & 0.008 & $1.24-4.10$ \\
\hline Used at least one opiate over 100 times & II7 (23.3) & 9.4 & 0.98 & 0.953 & $0.48-1.99$ \\
\hline Used cannabis over 100 times & 291 (57.9) & 10.3 & 1.23 & 0.494 & $0.67-2.29$ \\
\hline Used amphetamines over 100 times & $116(23.1)$ & 17.2 & 2.67 & 0.002 & $1.44-4.95$ \\
\hline Used cocaine/crack over 100 times & $96(19.1)$ & 16.7 & 2.34 & 0.010 & $1.23-4.48$ \\
\hline Used heroin over 100 times & $114(22.7)$ & 8.8 & 0.89 & 0.750 & $0.43-1.84$ \\
\hline \multicolumn{6}{|l|}{ Drug dependence } \\
\hline Cannabis dependence & $57($ (II.3) & 22.8 & 3.47 & 0.001 & I.7I-7.05 \\
\hline Amphetamine dependence & $72(14.4)$ & 18.1 & 2.48 & 0.010 & $1.24-4.96$ \\
\hline Cocaine/crack dependence & $94(18.7)$ & 21.3 & 3.68 & $<0.001$ & $1.97-6.87$ \\
\hline Heroin dependence & $110(21.9)$ & 10.9 & 1.21 & 0.582 & $0.6 \mathrm{I}-2.42$ \\
\hline Severe stimulant dependence & $59(9.9)$ & 28.8 & 6.24 & $<0.001$ & $2.86-13.63$ \\
\hline Severe opiate dependence & $107(21.3)$ & 11.2 & 0.69 & 0.388 & $0.30-1.59$ \\
\hline Severe cannabis dependence & $30(6.0)$ & 30.0 & 4.77 & $<0.001$ & $2.04-11.12$ \\
\hline Severe amphetamine dependence & $32(6.4)$ & 25.0 & 3.59 & 0.004 & $1.51-8.52$ \\
\hline Severe cocaine/crack dependence & $35(7.0)$ & 40.0 & 8.51 & $<0.001$ & $3.98-18.21$ \\
\hline Severe heroin dependence & $94(18.7)$ & 8.5 & 0.86 & 0.706 & $0.388-1.90$ \\
\hline
\end{tabular}

Functional psychosis was predicted strongly by severe cocaine dependence and severe cannabis dependence (adjusted OR for cocaine $=7.11, P<0.001$; adjusted $O R$ for cannabis $=3.26, P<0.05)$. Instead of drug dependence mediating all relationships between drug use and psychosis (as might be expected), other drug use measures were significant over and above any effect from drug dependence. For example, having used cannabis over 100 times had a marginally significant negative 
Table 2 Results from stepwise logistic regression: drug use variables with adjusted odds ratios for functional psychosis

\begin{tabular}{lccc}
\hline & Adjusted odds ratio & $P$ & $95 \% \mathrm{Cl}$ \\
\hline First amphetamine use before 16 years & 2.66 & 0.027 & $1.12-6.32$ \\
First cocaine use before I6 years & 2.83 & 0.035 & $1.08-7.43$ \\
Used cannabis more than I00 times & 0.46 & 0.060 & $0.21-1.03$ \\
Severe cannabis dependence & 3.26 & 0.023 & $1.18-9.03$ \\
Severe cocaine/crack dependence & 7.11 & $<0.001$ & $2.64-19.13$ \\
Severe heroin dependence & 0.31 & 0.027 & $0.11-0.88$ \\
Perceived difficulty of not smoking & 1.33 & 0.030 & $1.03-1.72$ \\
\hline
\end{tabular}

association (adjusted $\mathrm{OR}=0.46, P<0.06$ ) with functional psychosis when severe cannabis dependence was controlled. In other words, those who had used cannabis on over 100 occasions but did not reach the criteria for severe cannabis dependence were less likely to have psychosis. This apparent protective effect of having used cannabis at least 100 times is likely to be confounded with the effect of (absence of) severe cannabis dependence, because only two people who had not used at least 100 times were categorised as severely cannabis dependent. Overall, $30 \%$ of the severe cannabis dependence group had psychoses compared with $8.2 \%$ of those who were not severely dependent on the drug. Within the no-dependence group, $8.1 \%$ of those who claimed to have used cannabis less than 100 times were classified as having psychosis.

Although the amphetamine dependence measures did not reach significance in the model, use of this drug before the age of 16 almost tripled the likelihood of functional psychosis. A similar relationship was evident for early cocaine use (adjusted $\mathrm{OR}=2.83, P<0.05$ ) over and above the increased risk attributable to severe cocaine dependence.

In line with the bivariate relationship between severe heroin dependence and psychosis (which did not reach significance), a significant negative relationship was observed between these two variables when other drug dependencies were controlled. This indicates that individuals who reached the criteria for severe dependence on heroin were significantly less likely to have a classification of functional psychosis (adjusted $\mathrm{OR}=0.31, P<0.05$ ). There were too few cases to measure interaction effects between heroin and cocaine severe dependence even though 19 individuals reached the criteria for both.
Participants who reported that it would be difficult for them to go without smoking a cigarette for a whole day were slightly more likely to have a classification of functional psychosis (adjusted $\mathrm{OR}=1.33$, $P<0.05)$ when other drug use measures were controlled.

For all drug types examined, dependence needed to be severe to show a direct relationship with psychosis after adjusting for other drug effects. It should be noted that the data were analysed with respect to cocaine use and dependence in general, but the relationship between cocaine hydrochloride (or powder) and psychosis was consistently stronger than for crack cocaine. For example, when the logistic with severe crack dependence entered separately from severe cocaine powder depenand 11.3 , respectively.

\section{DISCUSSION}

\section{Characteristics of the sample}

The characteristics of the sample and levels of substance use involvement and dependence were broadly similar to those reported in other studies of the UK prison population (Gunn et al, 1991; Maden et al, 1991; Brooke et al, 1998). In particular, levels of psychosis were more than twenty times what has been reported from surveys of the general adult population (Meltzer et al, 1995). Similar (although less extreme) findings were reported by Brooke et al (1996). A previous paper reported high rates of dependence in both the prison and the homeless populations and also high rates of psychosis in the homeless population (Farrell et al, 1998). A substantial proportion of the sample reported that they had been homeless at some point in their regression model in Table 2 was re-run dence, the adjusted ORs for each were 2.7 lives and more than a quarter had spent time in local authority care. In the current sample, both were associated with higher risk of psychosis. The particularly high comorbidity between substance misuse and psychosis in the prison population provided the opportunity to examine associations between psychosis and dependence on specific drugs in this paper.

\section{Relationship between drug use, dependence and psychosis}

Strong relationships between drug use and psychosis were evident in the current sample. There is clear clinical evidence that amphetamines, cocaine and cannabis can result in an acute psychotic state that is indistinguishable clinically from other types of psychoses (Connell, 1958; Thornicroft, 1990; Unnithan \& Cutting, 1992; McGuire et al, 1994). There is a substantial literature that examines links between cannabis use and psychosis. However, despite the growth in cannabis use in the general population, no similar trends in the prevalence or incidence of psychosis have been observed. In addition, there is little to suggest that there has been a change in the average age at onset of psychosis, despite reasonable data indicating earlier cannabis initiation. In a longitudinal study of Swedish conscripts, a positive link between cannabis use and subsequent development of schizophrenia was noted (Andreasson et al, 1989). In contrast, Kwapil (1996) found that proneness to psychosis at baseline predicted substance misuse at follow-up, but no evidence for the reverse relationship (substance misuse predicting subsequent psychosis). Currently, the evidence suggests that cannabis can precipitate psychosis in people vulnerable to developing psychosis.

We found no evidence for a link between hazardous alcohol use or dependence and functional psychosis. Given previous reports that alcohol misuse is significantly related to increased risk of psychotic experiences in both men and women (Tien \& Anthony, 1990), this finding is contrary to expectations. However, it should be noted that data were not collected on patterns of early involvement with alcohol and early problematic drinking, which might correspond to our findings of increased risk associated with early initiation into cannabis, amphetamine, cocaine and heroin use. It is possible that such early use was associated with early and problematic use of alcohol. 


\section{Limitations}

A number of limitations to the study need to be acknowledged. Although the sample of prisoners was generated randomly, the extent to which the findings can be generalised to the wider population of adults in the UK is questionable. It is likely that the heavy drug involvement with its attendant criminality results in a larger drug-using sample in prison than would occur in a sample of people with psychosis drawn from the general population. Prison could, therefore, filter adults who are prone to both drug dependence and psychosis, thus increasing the observed effects. The prison sample could also be inclined to exclude cases where psychoses are non-drug related. Consequently, in the general population, the significant relationships between drug use and psychosis described here might be less prominent. The argument that the subjects found in this survey are not suffering from mental illness but simply manifesting the toxic or withdrawal effects of drugs has been examined in detail in a separate study comparing these with cases ascertained in the same way in the household (general) population (details available from the author upon request). This showed that delusions or hallucinations were clearly rated by the clinical interviewers on SCAN as not being attributable to drug effects in approximately three out of four prisoners.

\section{Early drug initiation and psychosis}

Table 1 shows that prisoners who first used cannabis, amphetamine, cocaine or opiates before the age of 16 are at greater risk of psychosis. Early drug initiation has been associated consistently with poorer longterm developmental outcome, higher rates of substance use and dependence and higher rates of psychiatric disorder (Ferguson et al, 1996). Although such outcomes might be expected in samples with psychiatric and social disturbance, it is possible that the drug use further augments the risk to an already vulnerable individual. However, the fact that we did not find a direct connection between psychosis and early cannabis use or early opiate use indicates that these links were explained by other drug use and dependence variables in the model. Nevertheless, early use of cocaine and amphetamines almost tripled the risk of psychosis in addition to the effect exerted by drug dependence. As the current study was cross-sectional, it is possible only to speculate on the nature of this link. One interpretation is that individuals with a predisposition to psychosis are more likely to use drugs at an early age. Alternatively, a period of special vulnerability to psychosis may exist.

\section{Drug dependence and psychosis}

In simple comparisons, the age of drug initiation for all drugs measured, the extent of stimulant use and dependence on cannabis, cocaine and amphetamines were linked significantly to functional psychosis. The logistic regression analyses indicated that the bivariate relationships between the majority of the drug-related variables and functional psychosis were actually explained by associations with severe drug dependence.

The fact that severe dependence on cannabis and cocaine were associated positively with psychosis, whereas for heroin the relationship was negative, suggests drug-specific effects. The relationship between psychosis and cannabis use has been widely documented (e.g. Hall, 1998; Tien \& Anthony, 1990) as has a similar relationship between amphetamine use and psychosis (e.g. Connell, 1958; Murray, 1998). However, although at the bivariate level amphetamine use was significantly related to functional psychosis, the current analyses did not support a relationship between amphetamine dependence and psychosis once the effects of other drug dependences were controlled. This is unlikely to indicate that the relationship between amphetamine use and psychosis has been overplayed. A possibility is that there is a strong association between cannabis dependence and amphetamine dependence and that the type of amphetamine commonly used (dexamphetamine) is often used in low concentration. This relationship might be very different if users were reporting consumption of high-purity methamphetamine, a similar drug that has recently gained popularity in countries such as Thailand (Farrell et al, 2002).

The belief that crack cocaine is more closely related to psychosis than dependence on cocaine powder was not supported by these analyses. This is difficult to explain, and there is no empirical literature comparing the risks between these substances. This issue merits further research. Overall, the current findings could suggest that, at least within prisoner populations, dependence mediates effects of other identifiable relationships with psychosis. In other words, increased risk of psychosis could be attributable to increased risk of drug dependence (within this high-dependence population).

In contrast, opioid and heroin dependence appeared to be linked to a reduced risk of psychosis. One interpretation of this finding is that the opioid class of drugs are not psychotogenic and therefore do not increase the risk of psychosis. It seems unlikely that the opioids have any antipsychotic effect other than a reduction in levels of arousal and amelioration of symptoms. The depressant effects of opioids could exacerbate affective symptoms as well as affective-type psychosis.

\section{Policy implications}

Severe dependence on cannabis and psychostimulants was associated with higher risk of psychosis whereas the opposite was true for severe dependence on heroin. The majority of substance misuse or addictiontype services in the UK deal predominantly with users who are opioid-dependent through the use of opiate agonist pharmacotherapies. Those presenting for treatment of cocaine, amphetamine or cannabis dependence comprise less than $10 \%$ of the overall activity reported to the Department of Health. However, our data suggest that these individuals are at greatest risk of psychosis and homelessness, and are generally more socially vulnerable.

\section{Treatment implications}

There are high levels of psychosis and high levels of drug dependence in the prison population. There is a need to develop and expand approaches to the management of such individuals and, in particular, to link them to appropriate types of community-based treatments on release from prison.

A possible treatment model is to provide structured psychosocial treatment delivered as part of a combined intervention between generic mental health services and community-based addiction services. Outcome studies indicate poorer results from drug-dependent individuals with psychiatric disorders. Experimental trials of such interventions are required in mental health settings if their application is to be fully developed.

Finally, it should be noted that much of the reported drug use and dependence was not linked significantly to psychosis. Only 
factors such as early initiation and severe dependence were related to increased risk. It is important that we do not overestimate the impact of drug use on psychotic disorders as we attempt to organise our services to respond to these complex problems.

\section{ACKNOWLEDGEMENTS}

We gratefully acknowledge the support of the Department of Health Drug Misuse Research Initiative, which supported this work. The views expressed in this paper are those of the authors.

\section{REFERENCES}

Andreasson, S., Allebeck, P. \& Rydberg, U. (1989) Schizophrenia in users and nonusers of cannabis. A longitudinal study in Stockholm County. Acta Psychiatrica Scandinavica, 79, 505-510.

Babor, T. F., de la Fuente, J. R., Saunders, J., et al (1992) AUDIT. The Alcohol Use Disorders Identification Test: Guidelines for Use in Primary Health Care. Geneva: World Health Organization.

Bohn, M. J., Babor, T. F. \& Kranzler, H. R. (1995) The Alcohol Use Disorders Identification Test (AUDIT): Validation of a screening instrument for use in medical settings. Journal of Studies on Alcohol, 56, 423-432.

Bridgwood, A. \& Malbon, G. (1995) Survey of the Physical Health of Prisoners, 1994. London: HMSO.

Brooke, D., Taylor, C., Gunn, J., et al (1996) Point prevalence of mental disorder in unconvicted male prisoners in England and Wales. BMJ, 313, 1524-1527.

\section{_ , Taylor, C., Gunn, J., et al (1998) Substance} misusers remanded to prison - a treatment opportunity? Addiction, 93, 1851-1856.

Claussen, B. \& Aasland, O. G. (1993) The Alcohol Use Disorders Identification Test (AUDIT) in a routine health examination of the long-term unemployed. Addiction, $\mathbf{8 8}$ 363-368.

Connell, P. (1958) Amphetamine Psychosis. London: Maudsley Monograph, no. 5. London: Chapman \& Hall.

Conigrave, K. M., Saunders, J. B. \& Reznik, R. B. (1995) Predictive capacity of the AUDIT questionnaire for alcohol-related harm. Addiction, 90, 1479-1485.

Farrell, M., Howes, S., Taylor, C., et al (1998) Substance misuse and psychiatric comorbidity: an overview of the OPCS National Psychiatric Morbidity Survey. Addictive Behaviors, 23, 909-918.

_ , Marsden, J., Ali, R., et al (2002)

Methamphetamine epidemic in South East Asia. Addiction, 97, 77I-772

Ferguson, D. M., Lynskey, M. T. \& Horwood, L. J. (1996) The short-term consequences of early onset cannabis use. Journal of Abnormal Child Psychology, 24, 499-512.

Gunn, J., Maden, A. \& Swinton, M. (1991) Mentally Disordered Prisoners. London: Department of Forensic Psychiatry, Institute of Psychiatry.

Hall,W. (1998) Cannabis and psychosis. Drug and Alcohol Review, I7, 433-444.

Kwapil, T. R. (1996) A longitudinal study of drug and alcohol use by psychosis-prone and impulsive-

\section{CLINICAL IMPLICATIONS}

- High rates of psychosis in the prison population, and high rates of drug dependence, should significantly impact on the management and outcome of psychosis in this setting and after release.

- Severe cocaine dependence has the strongest effect for risk of psychosis, which suggests a need to communicate to vulnerable people the risks associated with cocaine consumption.

- Much of the reported drug use and dependence was not linked significantly to psychosis and caution is urged when assessing the impact of drug use on psychotic disorder.

\section{LIMITATIONS}

- Respondents were drawn from the prison population and consequently the extent to which results could apply to the general adult population is unclear.

- Prison may filter adults who are prone to both drug dependence and psychosis and consequently the observed effects would be increased.

- Prison could possibly tend to exclude adults with psychoses relating to non-drug causes.

M. FARRELL, MRCPsych, A. BOYS, PhD, National Addiction Centre, London; P. BEBBINGTON, FRCPsych, Royal Free and University College Medical School, London; T. BRUGHA, MRCPsych, University of Leicester, Leicester; J. COID, FRCPsych, Forensic Psychiatry Research Unit, St Bartholomew's Hospital, London; R. JENKINS, FRCPsych, Institute of Psychiatry, London; G. LEWIS, FRCPsych, Division of Psychiatry, Cotham House, Bristol; H. MELTZER, PhD, Office for National Statistics, London; J. MARSDEN, PhD, National Addiction Centre, London; N. SINGLETON, MSc, Office for National Statistics, London; C. TAYLOR, MSc, National Addiction Centre, London

Correspondence: Michael Farrell, National Addiction Centre, 4 Windsor Walk, London SE5 8AF, UK. E-mail: m.farrell@iop.kcl.ac.uk

(First received I February 2002, revised 5 July 2002, accepted 31 July 2002)

nonconforming individuals. Journal of Abnormal Psychology, 105, 114-123.

Maden, A., Swinton, M. \& Gunn, J. (1991) Drug dependence in prisoners. BMJ, 302, 880

McGuire, P. K., Jones, P., Harvey, l., et al (1994) Cannabis and acute psychosis. Schizophrenia Research, I3, $16 \mid-167$.

Meltzer, H., Bill, B., Petticrew, M., et al (1995) OPCS Surveys of Psychiatric Morbidity in Great Britain, Report 1: The Prevalence of Psychiatric Morbidity Among Adults Living in Private Households. London: HMSO.

Murray, J. B. (1998) Psychophysiological aspects of amphetamine-methamphetamine abuse. Journal of Psychology, I32, 227-237.

Robins, L. N. \& Regier, D. A. (1991) Psychiatric Disorders in America: the Epidemiological Catchment Areo Study. New York: Free Press.

Singleton, N., Meltzer, H., Gatward, R., et al (1998) Psychiatric morbidity in England and Wales. A survey carried out in 1997 by the Social Survey Division of ONS on behalf of the Department of Health. London: Stationery Office.

_, Farrell, M. \& Meltzer, H. (1999) Substance Misuse among Prisoners in England and Wales. London: Stationery Office.

Thornicroft, G. (1990) Cannabis and psychosis. Is there epidemiological evidence for an association? British Journal of Psychiatry, 157, 25-33.

Tien, A. Y. \& Anthony, J. C. (1990) Epidemiological analysis of alcohol and drug use as risk factors for psychotic experiences. Journal of Nervous and Mental Disease, 178, 473-480.

Unnithan, S. B. \& Cutting, J. C. (1992) The cocaine experience: refuting the concept of a model psychosis? Psychopathology, 25, 7I-78.

World Health Organization (1992) SCAN: Schedules for Clinical Assessment in Neuropsychiatry. Geneva: WHO. 